Horizons philosophiques

\title{
Pour une pragmatique de la confession ou lorsque des monistes (Haeckel, Ardigò, Kaila) passent aux aveux
}

\section{Roch Duval}

Volume 10, numéro 1, automne 1999

Écritures et confessions

URI : https://id.erudit.org/iderudit/801109ar

DOI : https://doi.org/10.7202/801109ar

Aller au sommaire du numéro

Éditeur(s)

Collège Édouard-Montpetit

ISSN

1181-9227 (imprimé)

1920-2954 (numérique)

Découvrir la revue

Citer cet article

Duval, R. (1999). Pour une pragmatique de la confession ou lorsque des monistes (Haeckel, Ardigò, Kaila) passent aux aveux. Horizons philosophiques, 10(1), 85-105. https://doi.org/10.7202/801109ar d'utilisation que vous pouvez consulter en ligne. 


\title{
POUR UNE PRAGMATIQUE DE LA CONFESSION* OU LORSQUE DES MONISTES (HAECKEL, ARDIGÒ, KAILA) PASSENT AUX AVEUX
}

\begin{abstract}
$Y$ entonces, estremecido, enervado, retomo a la mesa y dudo ante las cuartillas de si un pobre hombre como yo, es decir, de si un pequeño filósofo, que vive en un grano de arena perdido en lo infinito, debe estampar en el papel los minúsculos acontecimientos de su vida prosaica...
\end{abstract}

Las confesiónes de un pequeño filósofo (Azorín) ${ }^{1}$

Bien que le terme "confession" apparaisse dans le titre de textes fonciers constituant le corpus de la philosophie occidentale, force est de constater que l'acception précise du vocable en question demeure, en règle générale, une énigme ayant su se dérober à la sagacité et à l'esprit d'analyse des philosophes. La confession, en tant que genre littéraire achevé, présente à celui qui désire percer son mystère et saisir les subtilités de son mécanisme interne deux difficultés majeures. Premièrement, comment faire complètement abstraction des connotations éthico-religieuses (saint Augustin) et esthétiques (Rousseau), si

* Allusion révérencieuse au texte de Darío Villanueva intitulé «Para una pragmática de la autobiografia" (Pour une pragmatique de l'autobiographie) dans Antonio Lara Pozuelo et al., La autobiografía en lengua española en el siglo veinte (L'autobiographie dans la langue espagnole au XXe siècle), Hispanica/Helvetica 1, Sociedad Suiza de Estudios Hispánicos, Lausanne, 1991, p. 201-218. À partir de maintenant, l'ensemble des traductions apparaissant dans le texte seront les miennes.

1. «Et alors, frémissant, harassé, je retournai à la table et hésitant devant les feuillets je demandai si un homme malheureux comme moi, c'est-à-dire si un petit philosophe vivant sur un grain de sable perdu dans l'infinité, devait inscrire sur le papier les événements anodins de sa vie prosaïque." Azorín (José Martínez Ruiz), Las confesiónes de un pequeño filósofo (1904) (Les confessions d'un petit philosophe), dans Obras Selectas de Azorín (Oeuvres choisies d'Azorín), Biblioteca Nueva, Madrid, $4^{\ominus}$ édition, 1969, p. 243-272., p. 246. 
fréquemment accolées au terme "confession"? Peut-on expliquer l'apparente désuétude du terme en invoquant une prétendue attraction osmotique, une interpénétration de différents champs sémantiques à l'oeuvre à une certaine époque, mais maintenant caducs et totalement inopérants? Notre univers, séculier et sur lequel plane l'esprit du nihilisme (et/ou du relativisme), a préféré substituer la pratique de l'autobiographie à celle de la confession. La tâche du philosophe, pour peu que celui-ci soit animé d'un souci d'exactitude et ait fait profession de foi de respecter une certaine correction linguistique, consiste précisément à distinguer la confession de l'autobiographie, car bien que ces pratiques littéraires présentent d'étonnantes ressemblances, elles ne forment cependant pas des entités gémellaires.

Deuxièmement, saint Augustin et Rousseau, tels deux astres évoluant dans le firmament de la philosophie occidentale, constituent en réalité d'immenses trous noirs exerçant une attraction telle que quiconque s'aventure dans leurs parages se sent irrémédiablement englouti et annihilé par la majesté de leur réalisation scripturaire. Les Confessions de saint Augustin inaugurent un style littéraire insurpassable; il faudra attendre Jean-Jacques Rousseau ${ }^{2}$ pour que l'embryon d'un «moi», à l'aube d'une subjectivité naissante, réussisse à se dépêtrer de la gangue dont le maintenait prisonnier l'univers théocentrique augustinien. Goethe dans Wahrheit und Dichtung, allant sur les brisées du "citoyen de Genève", parvint à s'arracher à l'orbite de Rousseau à prix d'or : dorénavant l'autobiographie supplante la confession. Dès lors, la confession semble appartenir à une autre époque et évoquer une autre sensibilité.

Le défi à relever consiste à disserter sur le style littéraire nommé «confession" sans "gloser" sur le corpus des commentaires, déjà impressionnant, dédiés à l'un ou l'autre de ces piliers de l'écriture confessionnelle que sont saint Augustin et Rousseau. L'emploi du terme "pragmatique", annon-

2. Jean Molino (1991) met toutefois en relief le rôle de relais joué par Pétrarque dans l'histoire de la confession et la fonction d'incubateur exercée par la Renaissance, en particulier par Jérôme Cardan, auteur du De Vita Propria. Cf. Jean Molino, "Interpretar la autobiografía» (traduction de G. Cantero-Oriol), dans Lara Pozuelo et al., op. cit, p. 107-138, p. 115. Voir du même auteur, "Stratégies de l'autobiographie au Siècle d'Or» dans L'autobiographie dans le monde hispanique, Paris/Aix en Provence, Université de Provence, 1980, p. 115-137. Guglielminetti qualifie l'entreprise de Pétrarque d' “imitazione agostiniana" (imitation augustinienne). Cf. Marziano Guglielminetti, Memoria e scrittura. L'autobiografia da Dante a Cellini. Piccola biblioteca Einaudi, Turin, 1977, p. 101. 
cé dans l'intitulé du présent essai, vise précisément à mettre en relief le caractère dynamique, ouvert, non dogmatique, heuristique, non hiératique, de la confession. Somme toute, peu m'en chaut de jouer le rôle d'un thuriféraire de saint Augustin, ou d'un laudateur servile de Rousseau. Mon intention est toute autre : j'aspire à dépoussiérer le style littéraire nommé confession en passant un coup de plumeau théorique afin de démontrer comment l'acte de confession demeure présent dans l'écriture philosophique contemporaine. ${ }^{3}$ Cette présence demeure cependant celée car elle ne se réduit pas à l'une ou l'autre des manifestations canoniques (saint Augustin/Rousseau) de la confession. II s'agit d'un type de confession dénommé "confession épistémologique" ou bien encore "confession pragmatique". Pour introduire cette nouvelle modalité dans l'ordre de la confession j'utilise un texte d'Ernst Heinrich Haeckel afin de démontrer comment la confession acquit une valeur heuristique et parvint même à constituer une pierre angulaire dans l'édification de la philosophie moniste.

On peut déduire comme corollaire la thèse suivante : L'autobiographie - pourtant enfant légitime de la Modernité - serait parvenue à remplacer la confession en amalgamant en un seul genre littéraire un style littéraire se déployant sur trois niveaux : la confession éthico-morale de saint Augustin (sphère du Bien), la confession esthétique de Rousseau (sphère du Beau) et finalement la confession pragmatique (sphère du Vrai). Faudrait-il attribuer à Goethe, l'auteur astucieux de Wahrheit und Dichtung, la formulation d'une conception de l'autobiographie où s'opérerait une procédure d'«indifférenciation", c'est-à-dire une disparition des différences entre les sphères du Bon, du Beau et du Vrai? ${ }^{4}$ Serait-ce au prix de cette

3. Le lecteur averti aura décelé l'allusion (ironique) faite à “Peut-on innover en autobiographie?" de Philippe Lejeune. Cf. Philippe Lejeune, "Peut-on innover en autobiographie?", dans L'autobiographie. Les Belles Lettres, Série Confluents psychanalytiques, 1988, p. 67-100.

4. Cf. Harald Grimen, "Programatisk rekonstruksjon av omgrepet "differensiering" " (Reconstruction programmatique du concept de "différenciation") dans David R. Doublet (dir.) Differensiering og rasjonalisering (Différenciation et rationalisation), Árbok 1987, Université de Bergen; Harald Grimen, "Problemet med dedifferensiering mellom vitskap, etikk og estetikk» (Le problème de l'indifférenciation entre la science, l'éthique et l'esthétique) dans Harald Grimen, Handling, differensiering og rasjonalitet. 12 forelesingar $i$ vitskapsteori. (Action, différenciation et rationalité. 12 conférences sur l'épistémologie) Senter for vitskapsteori skriftserie 10, Bergen, 1989, pp. 87-103; Harald Grimen, “Bør vi hápe pá at vitskap, moral og kunst nokon gang vil hange saman igjen?" (Pouvons-nous espérer qu'un jour la science, la morale et l'art soient de nouveau réunis?); dans Harald Grimen, Kritisk-konstruktive essays, Center for vitskapsteori skriftserie 15, Bergen, 1990, p. 169-200. 
indifférenciation que se serait consommée la confession? Dans cette hypothèse de travail, les rapports déjà imprécis entre la confession et l'autobiographie se présentent sous un nouvel éclairage. La vogue indéfectible de l'autobiographie proviendrait de cette disposition à amalgamer en un seul instant, ou en une seule écriture, les trois modalités de la confession: le Bon, le Beau et le Vrai. Or l'autobiographie n'agit pas de facto comme l'hyperonyme de ces trois modalités de la confession. Les problèmes conceptuels (définitionnels) éprouvés par l'autobiographie proviendraient vraisemblablement d'une incapacité à distinguer entre elles les sphères du Bon, du Beau et du Vrai, sphères pourtant perçues comme essentiellement distinctes depuis la Modernité. Le présent essai soutient qu'en phagocitant la confession l'autobiographie a fait disparaître du même coup la nécessité de discourir sur celle-ci. Or si les deux premières sphères de la confession possèdent leur archétype littéraire, leur parangon, la troisième sphère est toujours à la recherche d'un modèle exemplaire et idoine.

L'autobiographie, à la fois passage obligé et goulot d'étranglement de toute discussion sur la confession, sert de tremplin pour amorcer une discussion sur celle-ci. II s'agit de démontrer, dans un premier temps, comment l'autobiographie est parvenue à occuper une place si importante en philosophie. Dans un second temps on tente de distinguer formellement la confession de l'autobiographie en mettant à nu les attributs respectifs de chacun de ces genres littéraires. Finalement j'introduis un troisième type de modalité de la confession : la confession "pragmatique" ou "épistémologique". Le concept de vérité sert d'horizon à cette nouvelle modalité. II s'agit de s'interroger sur la nature de la confession ainsi dégagée.

\section{Les avatars de la tentation autobiographique}

Le philosophe tchèque Milic Capek ${ }^{5}$ notait, avec justesse, qu'il s'avère possible, voire même souhaitable, d'étudier l'oeuvre de la majorité des philosophes sans succomber à l'ultime indiscrétion consistant à passer au crible la vie privée de ceux-ci. Ce précepte, revêtant parfois un caractère quasi méthodologique, s'avère particulièrement judicieux dans bon nombre de cas. En vérité, la quête immodérée d'éléments croustillants de la vie d'un certain nombre de philosophes semble jouer en leur défaveur en jetant, rétrospectivement, une ombre délétère sur l'ensemble de leur production philosophique; mentionnons à titre d'exemple les cas affligeants et

5. Milic Capek, "The Presence of Masaryk's Thought", dans Milic Capek et Karel Hruby (dir.), T.G. Masaryk in Perspective. Comments and Criticism, Prague, SVU Press, 1981, p. 23-36. 
tristement célèbres des philosophes Martin Heidegger ou Giovanni Gentile ${ }^{6}$, entraînés et engloutis dans le maelström du fascisme, ou bien, plus récemment, le cas particulièrement pathétique du philosophe français Louis Althusser, en proie à une subite folie meurtrière. ${ }^{7}$

II appert toutefois qu'un nombre croissant de philosophes, faisant manifestement fi de l'admonition servie par Capek, se complaisent sciemment à agrémenter la trame de leur argumentation philosophique d'éléments autobiographiques. Le philosophe, à l'instar de tout autre créateur, se délecte à particulariser son oeuvre même si ses visées se veulent à l'origine objectives et universelles. Ainsi, il ne faut guère s'étonner de voir des philosophes consacrer autant de temps à échafauder d'austères théories et composer de doctes traités qu'à rédiger des journaux intimes, des mémoires, des autoportraits, leur autobiographie, voire même autoriser la publication de leurs échanges épistolaires, se livrant ainsi à des impudicités seyant mal à leur proverbiale objectivité.

Comment le phénomène de la confession est-il venu à se transformer en un acte aussi répandu que l'autobiographie de philosophes? La pléthore d'autobiographies de philosophes de tout acabit, déferlant d'une façon ininterrompue et croissante sur le marché du livre, exprime manifestement le symptôme d'un malaise, d'une crise patente d'identité de l'auteur. Faut-il voir dans l'émergence du style autobiographique un antidote à la dépersonnalisation qu'induit la circulation de masse des oeuvres littéraires? L'auteur arrive à éprouver le sentiment de se sentir dépossédé de son texte et se voit contraint à dresser un constat d'impuissance. Le philosophe tchèque Jan Fikacek (né en 1957) tourne en dérision cette sensation de dépersonnalisation en compensant ce sentiment de perte par l'ir.scription d'une surabondance de renseignements personnels visant à lidentifier, ou plus

6. Notons que parmi les derniers textes de Gentile figure une confession de type traditionnel (c.-à.-d. religieuse) "La mia religione" (1943) (Ma religion).

7. A ce propos voir l'excellent texte de Femando Savater où ce dernier examine sur un ton persifleur l'incidence de l'oxuricide d'Althusser sur la philosophie de ce dernier. Fernando Savater, "La tarea del héroe. Elementos para una ética trágica." (La tâche du héros. Éléments pour une éthique du tragique) dans Fernando Savater, La filosofía tachada. (La philosophie récusée), Barcelone, Editora Taurus, 1996. Dans une fascinante étude, Gisèle Mathieu-Castellani interprète les modalités du discours autobiographique en vertu du modèle judiciaire. L'autobiographie de Louis Althusser est autant une confession pro domo visant à expier une peine qu'un réquisitoire par lequel il se met lui-même en accusation. Cf. Louis Althusser, L'avenir dure longtemps. Suivi de Les Faits. Autobiographies, Paris, Stock/MEC, 1992; Gisèle Mathieu-Castellani, La scène judiciaire de l'autobiographie, Paris, Presses universitaires de France, 1996. 
précisément à le singulariser, en listant dans son C.V. une quantité superflue d'attributs physiques le caractérisant en propre. ${ }^{8}$ Ironiquement, on pourrait dire que de nos jours l'autobiographie, pour être complète, ne doit plus seulement relater l'histoire d'une vie, mais également narrer l'histoire d'un corps ${ }^{9}$ afin de compenser l'effet d'anonymat accompagnant la dépersonnalisation induite par l'écriture. Auparavant de tels problèmes conceptuels ne pouvaient survenir car le privilège de l'autobiographie était exclusivement réservé aux personnes menant des vies exemplaires et susceptibles de servir de modèles.

II est intéressant de souligner qu'à l'origine le genre littéraire des confessions était exclusivement réservé aux individus ayant acquis une incontestable notoriété ou jouissant d'un statut social particulier. Saint Augustin devint évêque d'Hippone en 396; n'eût été cette insigne ascension sacerdotale il est fort probable que les Confessions n'auraient jamais vu le jour. La singularité principale des Confessions de Rousseau provient principalement du fait qu'il rompt en quelque sorte avec cette exigence préliminaire que constitue la "pureté de haut lignage". Jean-Jacques, le roturier, le citoyen de "bas parage" et à qui rien ne réussit dans la vie, s'arroge le droit d'entrer au panthéon de la célébrité en prenant délibérément la plume. Ce faisant il relève le défi consistant à se dépeindre tel qu'il est, c'est-à-dire sans chercher à dissimuler les travers de sa personnalité. ${ }^{10}$ Le geste d'écriture équivaut chez Rousseau à un geste de création sui generis. L'écriture, pour celui qui avait été la cause du décès de sa mère (Suzanne Bernard) "en gésine", devient l'unique moyen de s'octroyer une identité et de crier raca à ses pourfendeurs. La mode était lancée; les philosophes n'avaient qu'à imiter Rousseau et croire à leur unicité.

Les Mémoires de l'artiste italien Benvenuto Cellini s'ouvrent d'ailleurs sur un appel à peine voilé invitant tous les philosophes à rédiger leur autobiographie :

8. Cf. http://pteryx.natur.cuni.cz/fikacek/ficacek.htm.

9. Cf. Richard Shusterman, "Soma und Medien" (Corps et médias), dans Gianni Vattimo/Wolfgang Welsch (dir.), Medien - Welten Wirklichkeiten, Munich, Wilhelm Fink Verlag, 1998, p. 113-126. Notons que H.G. Wells est conscient de cette dimension dans son autobiographie. Cf. H.G. Wells, Experiment in Autobiography : Discoveries and Conclusions of a Very Ordinary Brain (Since 1866), Londres, 1934.

10. “Ce n'est pas ce qui est criminel qui coûte plus à dire, c'est ce qui est ridicule et honteux." Jean-Jacques Rousseau, Les Confessions, Livre I, Paris, Ellipses, "Retour au texte", 1996, p. 13. 
Ceux qui ont connu dans leur vie une grande réussite ou quelque chose d'approchant devraient avec la sincérité de l'honnête homme écrire eux-mêmes leur autobiographie; mais sans s'attaquer à ce beau travail avant quarante ans. ${ }^{11}$

Pour peu que les philosophes se considèrent comme des hommes vertueux ou qu'ils soient dans la force de l'âge, la tentation de recourir à une forme quelconque de l'écriture du moi, de s'adonner à cette "tal bella impresa", acquiert une réalité indéniable au début du $19^{\mathrm{e}}$ siècle. C'est ce qui prélude à l'émergence d'une multitude de pratiques littéraires - toutes proches parentes de l'autobiographie - donnant l'occasion aux philosophes de s'exprimer d'une manière conforme à leur idiosyncrasie. L'appel fut certes entendu par Maine de Biran qui, sans pour autant délaisser l'écriture philosophique, entreprit la rédaction d'un journal intime. ${ }^{12}$

Genre littéraire moins étudié, mais non moins intéressant, les notes ou rapports de voyages si populaires au siècle dernier, constituent également un style littéraire offrant aux philosophes la possibilité de relater des événements personnels ou historiques, de se remémorer des rencontres fortuites ou préparées de longue date avec des confrères étrangers ou des gens du peuple, ou bien simplement de décrire en toute liberté des paysages, des lieux ou des phénomènes naturels, tout en privilégiant leur point de vue et en laissant parler leur for intérieur. ${ }^{13}$ Genre particulièrement cultivé chez les slaves, la formule de l'entretien [rozhovor] atteint un niveau inégalé de perfection dans la philosophie tchèque. ${ }^{14}$ Cette formule autorise volontiers les protagonistes du dialogue à adopter le ton de la confidence et, en outre, des révélations parfois surprenantes émergent au détour d'une question impromptue. La pratique de l'autobiographie philosophique débute dans le premier quart du $19^{\mathrm{e}}$ siècle. Le philosophe américain d'origine britannique, Alexander Bryan Johnson (1786-1867) succomba à la tentation de rédiger

11. Cf. Benvenuto Cellini, La vie de Benvenuto Cellini, écrite par lui-même. trad. Nadine Blamoutier, Paris, Éditions Scala, 1986, p.11.

12. Maine de Biran, Journal, Henri Gouhier, Neuchâtel, La Baconnière, 3 vol., 1954-1957.

13. Cf. "Minnen och reseanteckningar 1840" (Souvenirs et notes de voyages 1840 ) p. 507-535 et "Reseanteckningar $1841^{\text {" }}$ (Notes de voyages 1841) p. 584628 dans J.V. Snellman Samlade Arbeten II (Les oeuvres complètes de J.V. Snellman II), Helsinki, Statsrádets Kansli, 1992; “Tyskland. Skildringar och omdömen från en resa 1840-1841." (Allemagne. Descriptions et opinions sur un voyage 1840-1841) dans J.V. Snellman. Samlade Arbeten III 1842-1843, Statsrådets Kansli, Helsinki, 1993, p. 2-170.

14. Cf. Václav Belohradsky, Myslet zelen sveta. Rozhovor s Karlem Hvizdalou (Václav Belohradsky, Pensées sur l'écologie. Entretien avec Karl Hvizdala), Prague, Votobia, 1985, p. 3. 
son autobiographie. ${ }^{15} \mathrm{Au}$ cours du $19^{\mathrm{e}}$ siècle d'autres philosophes l'imitèrent : parmi ceux-ci mentionnons John Stuart Mill et Herbert Spencer. La pratique de l'autobiographie de philosophes constitue maintenant une pratique bien implantée, voire incontournable ${ }^{16}$.

Plus difficiles à cataloguer sont les journaux intimes de Ludwig Wittgenstein écrits en code secret. Grâce à la récente parution d'un livre norvégien ${ }^{17}$ il est maintenant possible de percer les arcanes de la personnalité complexe du philosophe viennois, aubain en terre norvégienne et horsain à Skjolden. Le fait de combiner la lecture de certains commentaires puisés à même ce journal intime à l'analyse de son échange épistolaire avec des résidents de Skjolden constitue une mesure heuristique indispensable pour sonder les profondeurs de la psyché wittgensteinienne.

Le cas de Wittgenstein s'avère particulièrement intéressant car cela met en relief l'attitude parfois contradictoire dont font preuve les personnes écrivant leur autobiographie ou, du moins, celles se laissant aller à des confidences. II existe d'une part un désir impérieux de parler de soi, de communiquer à l'univers tout entier ses sentiments les plus intimes, les plus secrets et, d'autre part, la tentation irrépressible de se taire, de garder pour soi ce qui nous appartient en propre. Ces sentiments contradictoires créent une tension incontournable où le désir de se dévoiler résiste opiniâtrement à la volonté de préserver son intimité. Dans un accès de pudibonderie, le regretté homme de lettres suédois Sven Delblanc ${ }^{18}$ regretta aussitôt l'envoi de son autobiographie chez l'imprimeur. L'attitude de Wittgenstein se révèle prisonnière de ce dilemme: à la fois écrire sur soi et interdire un accès trop facile, indécent, sur soi; d'où le subterfuge de l'écriture codée de son

15. Cf. Lars Gustaffson, "Det sista av sjuttonhundratalet” [Le dernier du $18^{\theta}$ siècle] dans Lars Gustaffson, Landskapets lángsamma förändringar. Essäer om människor och idéer. (Les lentes transformations du paysage. Essais sur les hommes et le idées), Stockholm, Natur och Kultur, 1992, p. 22-28.

16. La série The Library of Living Philosophers fait de l'autobiographie une priorité qu'elle inscrit dans la liste de ses principaux objectifs. Cf. "General Introduction to The Library of Living Philosophers", p. ix.

17. Knut Olav Amás et Rolf Larsen, Det stille alvoret. Ludwig Wittgenstein i Norge 1913-1950. (La tranquillité sereine. Ludwig Wittgenstein en Norvège 19131950), Postface de Kjartan Fløgstad, Oslo, Det Norske Samlaget, 1994. Voir également Kjell S. Johannessen, Rolf Larsen et Knut Olav Ámás (dir.), Wittgenstein and Nonway, Oslo, Solum Forlag, 1994; Stig Nystrand, Att leva sin självbiografi. Essäer om Ludwig Wittgenstein. (Vivre son autobiographie. Essais sur Ludwig Wittgenstein), Stockholm, Carlssons, 1997.

18. Cf. Sven Delblanc, Livets ax (L'épi de la vie) (1991) et Agnar (Balles de foin) (1993). Voir également Lars Ahlbom, Sven Delblanc, Natur och Kultur, Stockholm, Serie Litterära profiler, 1996. 
journal intime. L'autobiographie se situe donc à la jonction de deux univers opposés : le désir de se révéler et le souhait de demeurer celé.

Le désir de se révéler dénote une propension marquée au narcissisme. Le Suisse Henri Frédéric Amiel (1821-1881), un timide impénitent qui fut pourtant saisi d'une furor scribendi, se dévoila impudiquement dans un interminable journal, succombant pour ainsi dire à une véritable vésanie scripturaire. Plus près de nous, Paul Valéry se laissa également aller dans ses Cahiers à une véritable "graphorrhée». 19

La démonstration la plus éclatante de narcissisme, associée à une forme d'écriture du Moi, provient sans nul doute du poète finlandais Pentti Saarikoski (1937-1983). Ce dernier n'a-t-il pas maculé de son sperme les pages du manuscrit de son journal intime?20 Ici le désir de se dévoiler s'accompagne de la volonté explicite de se recréer. La fiction (la création d'un moi imaginaire porteur de sens) devient l'instrument par lequel l'auteur remplace, supplée, travestit, troque la réalité constituée par l'image d'un Moi évanescent et non substantiel — c'est-à-dire d'un Moi huméen ou nietzschéen n'offrant aucune unité au-delà de la simple succession des impressions - par un Moi substantiel et porteur de sens : "Minä teen elämästäni fiktiota, jotta se olisi totta21». Hyménée imparfaite, non consommée, entre le sperme de l'auteur et l'hymen de la page blanche, obstacle infranchissable qui demeure toujours un Autre pour le Moi de l'auteur; la quête de cette vérité substantielle du Moi, née de l'activité génératrice de fiction de l'auteur, engendre un Moi mort-né. Saarikoski se lance dans le conjungo avec la feuille de ses manuscrits en occultant le fait que sa semence, la seule véritable partie de lui-même qu'il laisse sur la feuille, ne pourra rien engendrer; seule l'encre - l'Autre fondamentalement (organiquement) différent de son Moi - pourra générer un discours sur lui. Prééminence de la poiesis sur la mimesis.

À ces diverses manifestations dénotant un narcissisme impudique s'oppose la fausse décence d'auteurs utilisant d'ingénieux artifices afin que leur Moi demeure voilé. Le recours à la dissimulation, au voile, convie d'un façon dialectique la possibilité du dévoilement. Divers artistes ont

19. Je propose d'introduire le néologisme "graphorrhée" - construit sur le modèle du terme logorrhée - pour caractériser un flot intarissable d'écriture et ne présentant aucune unité apparente.

20. Pentti Saarikoski, Nuoruuden päiväkirjat (Journaux de jeunesse), édité par Pekka Tarkka, Helsinki, Otava, 1984.

21. "Je fabrique ma vie comme une fiction, afin qu'elle soit vraie." Pentti Saarikoski (1984), op. cit., p. 25. 
succombé à la tentation de se voiler dans leur création. Le compositeur britannique Edward Elgar (1857-1934), incorpora dans ses Enigma Variations les initiales ou les surnoms d'amis proches de ce dernier. Ainsi, le finale E.D.U. renvoie à "Edoo" - déformation probable du prénom Edgard et que lui avait attribué son épouse. ${ }^{22}$ Que penser du subterfuge utilisé par l'auteur danois Svend Åge Madsen? L'intitulé d'un de ses plus récents romans, Syv aldres galskab (1994), se révèle un magnifique exemple de la dialectique s'instaurant entre le voile et le dévoilement. Le lecteur ignorant la langue danoise n'aura pas perçu que le titre du roman en question, une fois traduit en anglais, signifie "Seven Ages Madness". Ceci, comme on l'aura facilement observé, s'avère une approximation écrite du nom de l'auteur (Svend $\approx$ Seven, Åge $\approx$ Ages, Madsen $\approx$ Madness). Dans ce cas précis, le roman devient en quelque sorte un miroir dans lequel se mire l'auteur tout en ayant conscience que l'image ainsi reflétée est différente de l'objet réel.

Outre les raisons précédemment mentionnées, lesquelles font intervenir une dimension psychologique (lutte contre la dépersonnalisation), avancées pour expliquer l'explosion du style autobiographique chez les philosophes, il existe des motifs pratiques idoines à la philosophie. Comme nous l'avons signalé ci-dessus, la pratique de l'autobiographie s'explique en partie par l'attrait qu'elle exerce autant sur les contemporains que sur les générations futures en mettant en scène la vie de personnes vertueuses et jugées exemplaires; vies qu'il s'agit par la suite, idéalement, d'imiter ou d'intégrer en soi. À quel besoin peut donc répondre l'autobiographie de philosophes une fois escamotée l'attraction osmotique qu'exerce une vie jugée vertueuse ou digne d'être imitée?

L'autobiographie de philosophes répond à un besoin bien particulier et légitime et remplit une fonction précise. Cette fonction se traduit de la façon suivante :

\section{Fonction de l'autobiographie de philosophes}

L'autobiographie philosophique constitue un exposé condensé, abrégé, lapidaire, dont les principales fonctions sont:

a) de situer dans le vaste champ philosophique une filiation liant un

22. Cf. Livret du disque compact Polygramme 457-301-2. Elgar : Enigma/Holst : Les planètes. Texte français signé par Rémy Louis, trad. italienne par Roch Duval. 
disciple (l'auteur de l'autobiographie) à un/des maîtres(s), à une école, ou bien à un style défini.

b) l'explication diachronique de la genèse, des modifications, des ajustements, des rectifications, subies par une théorie ou un ensemble de théories ainsi que de son parachèvement ou, le cas échéant, des problèmes devant être résolus.

c) l'explication circonstancielle des palinodies de l'auteur considérées d'une façon rétrospective.

d) l'exutoire faisant office de règlement de compte, moyen de désavouer un maître ou une école. ${ }^{23}$

e) l'évaluation subjective de l'ensemble de l'oeuvre.

Nous avons pris conscience du rôle que joue l'autobiographie en philosophie et sous quelles formes cela se manifeste. II s'agit maintenant, d'une part, de formuler une définition générale de l'autobiographie et, ensuite, d'exposer en quoi la confession se distingue de ce style.

\section{Autobiographie et confession}

Le genre littéraire désigné par le terme "autobiographie» jouit actuellement d'une vague de popularité sans précédent. La notoriété du style ne doit cependant pas nous leurrer : l'autobiographie survient tardivement dans l'histoire de la littérature occidentale. ${ }^{24}$ Les historiens s'accordent pour établir le passage du XVIIIe au XIXe comme l'occasion rassemblant les éléments propices à l'éclosion du style autobiographique.

Bien qu'il ne faille pas négliger les critiques de Paul de Man et consorts $^{25}$, la nécessité d'une définition générique du style

23. Dans son autobiographie, Paul Feyerabend, qui fut pourtant à une époque un disciple féal de Popper, se complaît à dauber et morguer Sir Karl et se permet même le luxe d'occulter, voire de dénier cette influence. Les autobiographies respectives d'Axel Hãgerstrom et d'Adolf Phalén deviennent de mordantes philippiques où se livre un cruel combat afin de déterminer lequel des deux est le véritable fondateur de l'école d'Uppsala.

24. Laura Freixas affirme péremptoirement que cela constitue l'unique nouveau genre littéraire à émerger depuis Aristote. Cf. Laura Freixas, "Auge del diario ¿íntimo? en España", Revista de Occidente (Juillet-août 1996) No. 182/183, p., 5-14.

25. Paul de Man reproche la stérilité des discussions autour de l'autobiographie comme genre et maintient qu'empiriquement et théoriquement l'autobiographie ne se prête pas facilement à des définitions génériques. Cf. Paul de Man, "Autobiography as De-facement", ibid., The Rhetoric of Romanticism, Columbia University press, New York, 1984, p. 67-82. Cette idée est partagée, entres autres, par Avrom Fleischman Figures of Autobiography: The Language 
autobiographique se fait impérative. Georg Misch formule une définition succincte et laconique de ce genre littéraire : «Beschreibung des Lebens eines Einzelnen durch diesen selbst" (Description de la vie d'un individu par lui-même). ${ }^{26}$ Vague à souhait, la définition fourre-tout de Misch se révèle inapte à résoudre une armada de problèmes théoriques. Signalons un certain nombre de problèmes potentiels auxquels la théorie de Misch ne peut offrir une solution satisfaisante.

Considérons en premier lieu le terme "Beschreibung» (description). De quel type de description s'agit-il? On pourrait très bien imaginer un Doppelgänger27 (double/sosie) de Bill Gates, auquel on somme de rédiger une autobiographie, nous remettre un testament olographe dressant uniquement le bilan historique de ses biens matériels et de ses actifs. Totalement obnubilée par les succès financiers, réduisant pour ainsi dire sa vie à la simple poursuite et à l'acquisition de biens matériels, l'autobiographie du Doppelgänger de Bill Gates, toute aussi parcellaire et incomplète qu'elle soit, respecterait quand même les exigences listées dans la définition proposée par Misch. On pourrait également concevoir un Doppelgänger de la plantureuse Pamela Anderson, réduire son autobiographie à la description des différentes chirurgies plastiques (liftings, implants mammaires) qu'elle a subies. Or nous savons intuitivement que l'historique de l'ensemble des transactions bancaires/chirurgies plastiques effectuées par un individu ne peut en aucun cas constituer une autobiographie. Le genre autobiographique vise principalement la cohérence d'un récit rétrospectif d'où émerge une histoire porteuse de sens et non le simple alignement d'éléments hétéroclites (ou présentant une homogénéité contingente) dépourvus d'une signification d'ensemble. Nous pourrions dire que l'autobiographie doit avant tout être l'histoire d'une âme et non la simple juxtaposition fortuite d'événements ne présentant aucune cohérence entre eux.

of Self-Writing in Victorian and Modern England, Berkeley, University of California Press, 1983, p. 36.

26. Georg Misch, Geschichte der Autobiographie, Berne, 1949, Volume I, p. 7. Voir également Michael Jaeger, Autobiographie und Geschichte. Wilhelm Dilthey, Georg Misch, Karl Löwith, Gottried Benn, Alfred Döblin., Stuttgart, Verlag J.B. Metzler, 1995.

27. Sur l'utilisation du terme Doppelgänger et son utilisation possible dans une théorie de l'autobiographie voir le texte de Svend Age Madsen "Dobbeltgængeren og hans forgænger" (Le double et son prédécesseur), Gyldendals tidskrift Kritik, (novembre 1994). 
Pour qu'il s'avère d'une quelconque utilité, le terme "description» doit être davantage défini. II faut non seulement préciser plus finement la portée du terme "description" mais il faut également déterminer la modalité discursive de cette description. La définition de Misch demeure muette sur cet aspect. La description entrant dans le genre autobiographique ne peut être fantaisiste, poétique, symbolique, picturale; elle doit avant tout être conforme au modèle narratif afin qu'une trame historique s'y esquisse.

Deuxièmement, la philosophie analytique contemporaine nous a fréquemment mis en garde contre l'utilisation non qualifiée du terme individu (ou personne). Ce terme, qui nous renvoie à l'épineux problème de l'identité, constitue un élément devant être convenablement qualifié. La définition de l'autobiographie proposée par Misch fait précisément intervenir le terme «individu" sans toutefois le qualifier. Afin de démontrer l'importance que revêt la nécessité de préalablement définir le concept «individu" (Einzelnen) examinons le cas suivant. Imaginons un individu, que nous désignerons par $(X)$, ayant vécu une vie exemplaire, parangon de tempérance et de vertu, adulé et reconnu par ses pairs comme étant un homme de lettres possédant une prose impeccable et susceptible d'obtenir un jour le prix Nobel de la littérature. Sollicité par son éditeur, $(X)$ entreprend la rédaction de son autobiographie. La vie paisible de $(X)$ bascule un jour lorsque le destin lui joue le mauvais tour de mettre $(Y)$ sur son chemin. Ce dernier - un célèbre repris de justice, grimaud éthylique auquel un éditeur à également commandé une autobiographie - commet l'imprudence de conduire sa voiture en état d'ébriété. II frappe malencontreusement un piéton; on aura deviné que celui-ci est $(X)$. $(X)$ et $(Y)$ sont grièvement blessés et tous les deux nécessitent une délicate intervention chirurgicale. Par un hasard que l'on peut difficilement expliquer - sauf si c'est dans une Gedankenexpirement survenant dans un texte philosophique le chirurgien intervertit les cerveaux de $(X)$ et $(Y)$ : le cerveau de $(X)$ se retrouve dans le corps de $(Y)$ et vice versa. Une fois remis sur pied autant $(X)$ que $(Y)$ parachèvent leur autobiographie (sans pour autant relire ce qu'ils ont écrit auparavant). On peut facilement imaginer les problèmes théoriques qu'engendre une telle situation. L'autobiographie de $(X)$ est-elle celle de ( $X$ avant l'accident) ou bien celle d'un être hybride $(X Y)$ ? Pour résoudre de tels problèmes théoriques il s'avère impératif de postuler que le terme «individu» doit désigner une substance (quelconque) à laquelle on peut attribuer une continuité temporelle. La définition de Misch en ignorant 
de tels problèmes potentiels donne prise à des problèmes conceptuels de cet ordre. La nécessité de définir le concept "individu» acquiert une certaine urgence si on considère le cas (relativement) fréquent d'autobiographie où l'auteur se distingue du protagoniste du récit.

La faiblesse de la définition proposée par Misch réside en clair dans le fait qu'elle ne permet pas de suffisamment différencier l'autobiographie des autres styles littéraires, tels que la confession, le journal intime, les mémoires, la poésie autobiographique, le roman à la première personne, et qu'elle n'accorde pas assez d'importance aux problèmes potentiels découlant d'une incapacité de discourir sur le concept d'individu. En disant trop peu, elle ne dit rien d'intéressant.

Philippe Lejeune formule à mon avis une définition très intéressante de l'autobiographie :

Récit rétrospectif en prose qu'une personne réelle fait de sa propre existence, lorsqu'elle met l'accent sur sa vie individuelle, en particulier sur l'histoire de sa personnalité. ${ }^{28}$

Bien que la définition de Lejeune ne soit pas exempte de problèmes théoriques - nous pensons ici particulièrement aux problèmes liés au critère de sincérité que Lejeune érige en dogme dans sa notion de pacte autobiographique - celle-ci possède l'avantage de synthétiser en un tout cohérent les différentes pratiques autobiographiques proposées jusqu'à ce jour.

Disposant maintenant d'une définition générique (et programmatique) du style autobiographique, cherchons à formuler une définition théorique, ou au pis-aller opératoire, de la confession. Cette démarche définitoire se révèle nécessaire car autant la Confession de saint Augustin que les Confessions de Rousseau ne sauraient être réduites au style autobiographique pur et simple.

Disons dans un premier temps que la confession se distingue de l'autobiographie dans sa dimension temporelle. Tandis que la dimension temporelle mise en jeu par l'autobiographie se caractérise par la cohérence et la continuité de la trame narrative du récit où l'on postule la présence d'un développement ininterrompu allant grosso modo de l'enfance à lâge adulte, la dimension temporelle introduite par la confession porte le sceau d'un rupture entre deux moments irréconciliables. La confession est la

28. Philippe Lejeune, "Le pacte autobiographique" dans Le pacte autobiographique, Paris, Éditions du Seuil, 1975, p. 13-45., p. 14. 
perception d'une césure radicale entre une période antérieure où l'auteur se perçoit comme dévoyé et une période postérieure où il se juge rédimé. La perception de cet infranchissable abîme n'entre jamais dans la quiétude de la mémoire où il pourrait reposer et finir par se déliter avec le temps; la confession demeure une cruelle plaie vive que le temps ne pourra cicatriser. L'auteur japonais Mishima Yukio (La confession d'un masque) le saura à ses dépens : seul le suicide viendra réparer la honte du dévoilement.

Deuxièmement, si l'autobiographie révèle l'histoire d'un individu en devenir, la confession célèbre la naissance d'un nouvel individu. Personne ne sort indemne d'une confession. Au terme de cet hiatus, le "confessé" répudie celui qu'il était auparavant. Le poète suédois Bertil Malmberg exprime dans ces merveilleuses strophes ce sentiment d'étrangeté et d'altérité vis-à-vis de soi-même qui s'installe chez celui qui se confesse : "Jag ser den ynling som en gång var, och förstår att jag aldrig skall bli hans vän (Je vois le jeune homme que je fus autrefois, et comprends que je ne deviendrai jamais son ami)".

Après avoir écrit sa Confession saint Augustin ne fut jamais le même. Les Confessions du bon Jean-Jacques font office de certificat de baptême : en écrivant ses Confessions il met au monde un être différent, un être bonifié, un être dont il dégage patiemment la véritable nature afin de défier et combattre l'image pervertie que ses détracteurs veulent véhiculer de lui.

Troisièmement, l'horizon dans lequel se meut la confession en est un de crise. C'est pourquoi la confession ne peut être un récit en prose mais revêt davantage la forme d'une plainte. Quatrièmement, si l'autobiographie constitue une somme d'événements auxquels on attribue a posteriori une signification, la valeur de la confession réside a priori dans un événement unique facilement identifiable mais dont la présence se fait toujours sentir et dont le rythme berce le quotidien du confessé. Ceci veut dire que les confessions portent le signe de leur naissance comme une stigmate: saint Augustin peut identifier le moment précis de sa conversion que tente de faire revivre sa confession, soit en août 386. Le Discours de la méthode de René Descartes, que nous pouvons considérer d'une certaine manière comme une confession, s'inscrit dans une temporalité précise : la nuit du 10 au 11 novembre 1619. Le bois de Vincennes, une nuit d'octobre 1749 sert de cadre référentiel à la confession de Rousseau. ${ }^{29}$

29. Cf. Karl J. Weintraub, "Autobiography and Historical Consciousness" dans Critical Inquiry 1 (1975), p. 821-848. 
Nous sommes actuellement en mesure d'énoncer une définition générale de la confession:

Confession : Acte de langage ponctuel, identifiable à un instant précis, par lequel un agent réel marque une distance envers son moi perverti pour se réfugier dans un moi régénéré et purifié consubstantiel à la découverte d'une vérité qui ne le quittera plus le reste de sa vie et qui sert de point d'ancrage à l'édification d'une théorie ou qui explique une attitude intellectuelle donnée.

II nous reste maintenant à illustrer cette définition à l'aide d'un exemple pratique qui en plus de confirmer cette lecture présente une troisième modalité de la confession, différente de celles de saint Augustin et Rousseau : la confession pragmatique d'Ernst Haeckel.

\section{Étude de cas : Le monisme mesuré à l'aune de la confession}

Le monisme philosophique constituait à la fin du siècle dernier une théorie philosophique alliant les plus récentes découvertes scientifiques à une théorie métaphysique vouée à la réconciliation de paires d'éléments faussement identifiées comme distincts : être et pensée, corps (Körper) et conscience, corps (Leib) et âme, esprit et nature, force et matière, unité et multiplicité, essence et apparence, Dieu et monde, et ainsi de suite ${ }^{30}$. Cette idée-force eut de nombreux propagateurs en Allemagne (Lotze, Eucken, Haeckel, Mach, Avenarius, Eduard von Hartmann et tutti quanti) et dans de nombreux pays européens (Høffding, Spencer, Westermarck, Rein, Neiglick, Lagerborg, Ardigò, Carus [aux États-Unis]).

Le 9 octobre 1892, à Altenburg, Ernst Haeckel (1834-1919), une des chevilles ouvrières du monisme philosophique, présenta une allocution afin de propager la conception moniste de l'univers. L'attrait que présente cette conférence (Der Monismus als Band zwischen Religion und Wissenschaff) réside principalement dans la confession qu'y prononce Haeckel. ${ }^{31}$ L'intérêt de la confession de Haeckel ne consiste pas tant dans le caractère abstrait

30. Pour un exposé complet sur le type de monisme de Haeckel voir de Wilhelm von Schnehen, "Haeckels "Reiner" und "Konsequenter" Monismus" (Le monisme pur et conséquent de Haeckel) dans Arthur Drews (dir.), Der Monismus dargestellt in Beiträgen seiner Vertreter, Jena, Eugen Diederich Verlag, 1908, p. 102-148

31. L'intitulé de l'édition anglaise met en exergue le moment de la confession en traduisant le titre de l'opuscule de Haeckel par The Confession of faith of a Man of science. Cf. Ernst Haeckel, The Confession of faith of a Man of science, trad. de J. Gilchrist, Londres, Adam and Charles Black, 1894, $19034^{e}$ édition. J'utilise un fac-similé (1973) produit par l'Université du Michigan à Ann Arbor. 
qu'elle revêt que dans la fonction qu'elle est appelée à jouer dans son argumentation. En effet, Haeckel demeure laconique lorsqu'il s'agit de décrire l'aspect existentiel de la confession. Sauf une rapide allusion à un type de rencontre (physique et théorique) déterminante pour Haeckel ${ }^{32}$ on cherchera en vain une description détaillée de cet événement charnière. Dans un langage scientifique et dépouillé Haeckel sollicite le lecteur à accepter la confession non pas comme un acte de foi aveugle mais comme une déduction revêtant une grande probabilité. En d'autres termes Haeckel ne peut prouver d'une façon apodictique que la substance moniste existe véritablement mais les développements de la science sont tels que le passage de la certitude des prémisses à la conclusion inférée ne doit faire aucun doute dans l'esprit des hommes. La confession devient donc un moment argumentatif visant à assurer la validité d'une conclusion nécessaire au maintien de la cohérence du système. La confession vient ajouter à la probabilité objective des faits scientifiques la probabilité subjective d'un principe foncier nécessaire à la validation du système que l'on désire introduire. Le garant de la philosophie moniste de Haeckel n'est plus un Dieu personnel mais une idée incarnée de la vérité. ${ }^{33}$ Ce faisant Haeckel indique que la vérité scientifique devient l'objectif auquel on doit tendre et il récupère du même coup dans sa philosophie moniste les trois éléments essentiels de la vie: Vérité, Beauté et Bonté. ${ }^{34}$

La "confession" de Haeckel n'a en soi rien de spectaculaire. Au laconisme teuton du philosophe et biologiste allemand il faut opposer la prolixité méridionale du philosophe italien Roberto Ardigò (1828-1920). Le philosophe mantouan rédigea à plusieurs reprises et à diverses époques son autobiographie philosophique. ${ }^{35}$ Bien qu'Ardigò relata maintes fois son apostasie (survenue vers 1869), signifiant du même coup sa conversion au positivisme, c'est un Ardigò quasi octogénaire qui narre d'une façon

32. "Darwin opened our eyes", Haeckel op. cit., p. 73.

33. "We may substitute for a personal God the personified idea of truth". Haeckel, op. cit., p. 77.

34. "Monistic investigation of nature as knowledge of the true, monistic ethic as training for the good, monistic aesthetic as pursuit of the beautiful - these are the three great departments of our monism...". Haeckel op. cit., p. 86.

35. Succinta narrazione della mia vita scientifica (Brève narration de ma vie scientifique) (1877), appendice I dans Wilhelm Büttermeyer, Roberto Ardigò e la psicologia moderna. Florence, La Nuova Italia Editrice, 1969, p. 89-93; La morale dei positivisti (1878-79) (La morale des positivistes) dans Roberto Ardigò, Opere filosofiche I, Padoue, Draghi, $3^{\theta}$ éd. 1929; Ibid., Guardando il rosso di una rosa (1907) (Contemplant le rouge d'une rose), Opere filosofiche $X$, Padoue, Draghi, 1909, p. 241-258. 
poignante cette confession. L'écrit autobiographique Guardando il rosso di una rosa (1907) mérite véritablement le titre de confession car la description qu'Ardigò fait de cet événement regroupe un grand nombre d'éléments rhétoriques venant donner corps au récit de sa conversion et de sa découverte de la vérité du monisme. Est-ce un hasard si Ardigò, à l'instar de saint Augustin, situe sa conversion dans un jardin? On sait que Rousseau s'était amusé à reproduire et adapter certains éléments de la confession de saint Augustin. Faut-il croire que le positiviste italien a succombé à cette même tentation? Ou bien succombe-t-il à une influence larvée instillée dans son esprit par son ami Gaetano Trezza (1827-1892), auteur des Confessi di uno scettico? (Confessions d'un sceptique). Quoi qu'il en soit, Ardigò nous relate là une véritable confession car les éléments essentiels de ce genre littéraire s'y retrouvent : désignation et rejet d'un passé dévoyé et découverte de la vérité à un endroit et un moment déterminés. La découverte de cette vérité porte un nom et un lieu : il s'agit de la lecture d'un livre du physiologiste Johannes Müller dans un jardin en 1869. Pourquoi Ardigò a-t-il attendu si longtemps (1907) pour relater un tel événement (1869)? II est évident que l'appel à une confession ajoute du crédit (probabilité subjective) à la démonstration d'une vérité que l'on voudrait objective, mais qui malgré toute la bonne volonté du monde demeure indémontrable. La confession ne serait-elle rien d'autre qu'un artifice littéraire, comme chez Haeckel, jouant un rôle précis dans l'argumentation philosophique (instiller une "vérité" que l'on ne peut démontrer d'une manière apodictique)? Cette hypothèse semble intéressante si on considère la confession d'un autre moniste, le Finlandais Eino Kaila (1890-1958).

Dans un texte important, dans lequel interviennent des éléments autobiographiques présentés sous le ton de la confession, Eino Kaila narre l'expérience intime présidant à l'origine de son éveil philosophique. C'est en 1953, alors âgé de 63 ans, que Kaila se confesse en révélant une expérience intime à la base de sa vision moniste de l'univers :

Je me souviens de ce jour où se produisit mon éveil philosophique. C'était au mois de juillet, un jour clair d'été, alors que j'étais étendu au fond d'un canot, au large d'un lac d'une beauté sauvage, et me laissais bercer par les vagues et que je poursuivais du regard la course de cumulus estivaux dans le ciel. (...) Mon éveil philosophique se produisit au moment où je saisis que tout ce qui existe est dans un sens profond un tout unifié, pour ainsi dire une "unité englobante", un univers se structurant de lui-même. ${ }^{36}$ 
Même si Kaila était un farouche opposant au monisme de Haecke/ ${ }^{37}$ et qu'il ne connaissait probablement pas Ardigò, nous retrouvons cependant le même type de confession; confession qui est appelée à jouer un rôle foncier dans l'instillation d'une vérité. Cette pratique est d'autant plus étonnante si on considère le cas d'un réputé philosophe contemporain, protagoniste d'une forme de matérialisme émergent, ${ }^{38}$ recourir lui aussi à la confession afin d'expliquer les idées à la base de son projet philosophique. ${ }^{39}$ Niiniluoto relate les circonstances de son éveil philosophique en faisant implicitement appel à l'image de la "révélation lacustre" de Kaila. Dans un court essai intitulé Tietämättömyyden rantaviivalla il reprend à son compte la confession de Kaila : "Je peux représenter mon propre éveil philosophique de la façon suivante : cela se produisit à Vaasa le 10 juillet $1965 .{ }^{40 \text {, }}$

S'agit-il ici de l'expression véritable de l'expérience vécue par un jeune Niiniluoto, alors à peine âgé de 19 ans, ou bien avons-nous plutôt affaire à une confession revue et ajustée et conviée à jouer un rôle précis dans une argumentation? Envers qui ou quoi Niiniluoto est-il le plus fidèle? Envers ses souvenirs ou bien envers une filiation symbolique avec la cheville-ouvrière de la philosophie finnoise? Quelle est, somme toute, la part du vécu et la part de la fabulation volontaire, téléguidée, dans cette réminiscence? Ces divers exemples ne nous incitent-ils pas à méditer davantage sur la valeur réelle ou artificielle du style "confession" en philosophie?

36. Eino Kaila, "Laatujen asema suureiden maailmassa" (La place des qualités dans un monde de quantités), Valvoja 73 (Le protecteur), p. 261-275. Texte repris dans Eino Kaila, Valitut teokset 2. 1936-1958 (Eino Kaila. Oeuvres choisies 2. 1936-1958), Ilkka Niiniluoto (dir.), p. 505-524; p. 505. Le souligné est dans le texte.

37. Georg Henrik von Wright, "Eino Kaila's Monism" dans Ilkka Niiniluoto et al., Eino Kaila and Logical Empiricism, Acta Philosophica Fennica 52, Helsinki, Societas Philosophica Fennica, 1992, p. 71-91.

38. Cf. llkka Niiniluoto, Maailma, Minä ja Kulttuuri, (Monde, Moi et culture), Helsinki, Otava, 1990.

39. Dans le cas de Niiniluoto il s'agit d'une forme de réalisme critique.

40. Ilkka Niiniluoto, «Tietämättömyyden rantaviivalla" (Sur le littoral de l'ignorance), dans Ilkka Niiniluoto, Tiede, filosofia ja maailmankatsomus. Filosofifia esseitä tiedosta ja sen arvosta. (Science, philosophie et vision du monde. Essais philosophiques sur la science et sa valeur), Helsinki, Otava, 1984, p. 13-18. 


\section{Conclusion}

Le terme confession apparaît plusieurs fois dans l'intitulé d'ouvrages philosophiques. II n'existe malheureusement pas de définition précise de ce terme. Dans cet essai j'ai tenté brièvement d'indiquer quelle est la valeur sémantique de ce terme en retraçant son histoire et en fixant les paramètres de son application. Nous avons mentionné qu'il existe deux types bien connus de confession; la confession de saint Augustin et celle non moins célèbre de Jean-Jacques Rousseau. Guidé par l'idée que ces deux types de confession renvoient respectivement à deux manières bien précises de concevoir la réalité (sphère du Bon/sphère du Beau) nous avons cherché une troisième forme de confession où serait à l'oeuvre une conception de la réalité éclairée par le concept du Vrai. Le qualificatif "pragmatique" désigne d'une part le rôle actif qu'est appelée à jouer une telle conception de la confession dans l'érection de la philosophie moniste et d'autre part le caractère ouvert, non dogmatique d'une telle entreprise. Une confession de type pragmatique, ou épistémologique, survient lorsqu'un philosophe introduit dans la trame du récit de sa vie un épisode ponctuel, où se produit un événement décisif quant à la formation, ou le cas échéant à la poursuite, d'une démarche philosophique déjà ébauchée ou sur le point de se constituer. Bien que ce qui est désigné par la confession joue un rôle foncier nous serions plutôt porté à considérer cela comme une sorte de "décisionnisme", 41 obéissant à l'impératif constructiviste de présenter une théorie cohérente.

La confession pragmatique remplit une fonction bien précise : dans l'impossibilité de fonder d'une façon absolue la validité de prémisses essentielles au développement d'une théorie donnée, la confession sert d'argument post hoc pour introduire une telle prémisse manquante. Une confession pragmatique constitue d'une certaine manière un aveu d'impuissance.

À linstar de la confession de saint Augustin et celle de Rousseau, des problèmes conceptuels minent à la base la confession pragmatique. Si on peut douter à juste titre que l'image que Rousseau présente de lui-même est véritablement l'image de son moi naturel, on peut également mettre en

41. Cf. Peter Janich, "Voluntarismus, Operationalismus, Konstruktivismus. Zur pragmatische Begrūndung der Naturwissenschaften", dans ibid., Konstruktivismus und Naturerkenntnis. Auf dem Weg zum Kulturalismus, Francfort, Suhrkamp, 1996, p. 8-21. 
doute le caractère véridictif de la confession pragmatique. Le principal problème concerne la nature même du concept de vérité. 42

Empruntant une image chère à l'homme de lettres uruguayen Mario Benedetti 43 nous pourrions dire que la confession pragmatique, telle l'échelle de Wittgenstein, constitue une sorte d'échafaudage dont on devrait se débarrasser une fois l'ouvrage accompli.

\author{
Roch Duval \\ Université de Montréal
}

42. Cf. Jaroslav Peregrin (dir.), The Nature of truth (if any). Proceedings of the International Colloquium, Prague, September 17-20, 1996, Prague, Filosofia, 1997.

43. Benedetti intitule son plus récent récit autobiographie Andamios (Échafaudages), Montevideo, Seix Barral, 1996. 